Hence, a quadrature shows that $\phi(t) / t \rightarrow \infty$, as $t \rightarrow \infty$. In view of $x(t)=r(t) \cos \phi(t)$, this implies the truth of (9) for the number of the zeros of $x(t)$.

It follows in the same way that

$$
N(t) / t>\text { const. }>0 \quad \text { as } \quad t \rightarrow \infty,
$$

if every solution of (1), instead of satisfying (8), remains just bounded as $t \rightarrow \infty$. Similarly, if $x(t) / t^{1 / 2}$ remains bounded for every solution of (1), then (1) must be oscillatory, since (2) then follows in the same way as (9) and (10) did.

\title{
THE DISTRIBUTION OF PLANE ANGLES OF CONTACT*
}

\section{BY J. WOLFOWITZ (Columbia University)}

Let $R=\omega(\theta)$ be a closed, simple, convex, plane curve, with a continuous tangent and the origin in its interior, and with $R, \theta$ its polar coordinates. A particle $A$ moves in a straight line at constant speed $k$ from time $t=0$ to $t=T$. Another particle $B$ moves with unit speed in a straight line coplanar with the path of $A$. The probability that at time $t=0$ the particle $B$ will be in any sub-region $g$ of a sufficiently large region $G$ containing the starting point of $A$ (at time $t=0$ ) is proportional to the area of $g$. The probability that the azimuth of $B$ 's motion will lie between the angles $\alpha, \beta$, is $(\beta-\alpha) / 2 \pi$ and is independent of the starting position of $B$. Let $\theta$ be the angle from the direction of $A$ 's motion to the particle $B$, and $R$ the distance from $A$ to $B$. When $R=\omega(\theta)$ a "contact" is said to occur. Let $\phi$ be the angle from the radius vector from $A$ to $B$ to the direction of $B$ 's motion. The couple $(\theta, \phi)$ characterize a contact. Naturally no contact need ever occur between $t=0$ and $t=T$, or at any other time, for that matter. In this note we shall be concerned with the probability distribution of $(\theta, \phi)$ in the totality of contacts which $d o$ occur between $t=0$ and $t=T$, and excluding the possibility that, at $t=0, R<\omega(\theta)$. We will show that the probability density $f(\theta, \phi)$ of $(\theta, \phi)$ is $c\left(D^{*}+\left|D^{*}\right|\right)$ where

$$
D^{*}=-\omega(\theta) \cos \phi+\frac{d \omega}{d \theta} \sin \phi+k\left[\omega(\theta) \cos \theta+\frac{d \omega}{d \theta} \sin \theta\right]
$$

and $c$ is a constant defined by

$$
\int_{0}^{2 \pi} \int_{0}^{2 \pi} f(\theta, \phi) d \theta d \phi=1 .
$$

The curve $R=\omega(\theta)$ may be thought of as a field of force surrounding the particle $A$. If $A$ and $B$ are circular disks and $\omega(\theta)$ identically equal to the sum of their radii, a "contact" would be literally such. The following should also be noted:

a) The result is independent of $T$. The requirement that $G$ should be sufficiently large means that $G$ should contain a circle, centered at the position of $A$ at $t=0$, and of radius sufficiently large (the minimum radius depends upon $T$ ). Once $G$ is sufficiently large, $f(\theta, \phi)$ does not depend upon $G$.

b) The result depends only upon the ratio $k$ of the speeds. The constant $c$ depends

*Received July 13, 1948. 
upon $k$, in general. If $k$ is a chance variable distributed between $k_{1}$ and $k_{2}$ with probability density $m(k)$, the probability density of $(\theta, \phi)$ is then

$$
\int_{k_{1}}^{k_{2}} f(\theta, \phi) m(k) d k
$$

(The minimum size of $G$ then depends not only on $T$, but also on $k_{2}$ ).

c) The result is valid when $A$ is at rest $(k=0)$.

d) The result holds without change if the azimuth and initial position of $A$ are chance variables with any distributions whatever, provided only that they be independent of the initial position and azimuth of $B$.

e) When $\omega(\theta)$ is identically constant the parameter $c$ may be evaluated without difficulty in terms of Legendre's elliptic integrals of the first and second kind.

f) When $A$ is at rest and $\omega(\theta)$ is a constant, $f(\theta, \phi)$ is proportional to $\cos \phi$ when $\pi / 2<$ $\phi<3 \pi / 2$, and is zero otherwise.

g) The method of proof is applicable to the problem of contacts in space, except that the computations become much more involved.

We now proceed to the proof.

The direction of motion of particle $A$ being $\theta=0$, the direction of motion of particle $B$ when its contact is characterized by $\theta, \phi$ is $(\theta+\phi)$. Let $x, y,(\phi+\theta)=\psi$, be, respectively, Cartesian coordinates of position and the azimuth of the direction of motion of particle $B$ at time $t=0$. The origin of the Cartesian system will be placed at the position of $A$ at $t=0$ and the positive $x$-axis in the direction $\theta=0$. Let $H$ be the rectangle in $\theta, \phi$ space whose vertices are $(\theta, \phi),(\theta+\Delta \theta, \phi),(\theta, \phi+\Delta \phi)$ and $(\theta+\Delta \theta$, $\phi+\Delta \phi)$, respectively. Then the probability $\Pi$ of a contact characterized by a point in this rectangle $H$ is a constant multiplied by the volume of a region $S$ in $x, y, \psi$ space. This region contains those (and only those) points $x, y, \psi$ which are such that, if the position and motion of particle $B$ at time $t=0$ are described by $x, y, \psi$, a contact in $H$ will occur within $0 \leq t \leq T$. The desired probability density $f(\theta, \phi)$ is given by

$$
f(\theta, \phi)=\lim _{\substack{\Delta \theta \rightarrow 0 \\ \Delta \phi \rightarrow 0}} \frac{\Pi}{\Delta \theta \Delta \phi} .
$$

We shall now show that every section of $S$ cut by a plane $\psi=$ constant is, to within infinitesimals of order higher than the first, a parallelogram. Let a contact $\theta, \phi$ occur at time $t, 0 \leq t \leq T$. Then at time $t$ the position of $A$ will be $(k t, 0)$, the position of $B$ will be $(k t+R \cos \theta, R \sin \theta)$. The position of $B$ at $t=0$ was

$$
(k t+R \cos \theta-t \cos \psi, R \sin \theta-t \sin \psi) .
$$

Hence, in order to have a contact $(\theta, \phi)$ the particle $B$ must be, at $t=0$, on a line running from

$$
M=(R \cos \theta, R \sin \theta)
$$

to

$$
M^{\prime}=(R \cos \theta+k T-T \cos \psi, R \sin \theta-T \sin \psi) .
$$

Let now $\theta, \phi$ move in the rectangle $H$ along the line $\psi=$ constant. Both $M$ and $M^{\prime}$ are displaced by

$$
\delta\left(-R \sin \theta+R^{\prime} \cos \theta, R \cos \theta+R^{\prime} \sin \theta\right)=(c, d)
$$


where $\delta=\delta \theta=-\delta \phi$, and $\delta \theta$ and $\delta \phi$ are the increments of $\theta$ and $\phi$, respectively. (Here $R^{\prime}=d \omega(\theta) / d \theta$.) Thus the parallelogram has vertices

$$
M, M^{\prime}, M+(c, d), M^{\prime}+(c, d) \text {. }
$$

Its area, to within infinitesimals of order higher than the first, is $D \delta$, where $D$ will be evaluated shortly. $D$ is constant in $H$.

Let $\delta(\psi)$ be the maximum difference between any two values of $\theta$ which are both in $H$ and lie on a line $\psi=$ constant. Then

$$
\int_{H} \delta(\psi) d \psi
$$

is proportional to the area of $H$. Thus $f(\theta, \phi)$ is proportional to $D$, the proportionality constant being independent of $\theta$ and $\phi$.

As for $D$, it is the absolute value of the vector product of the vector $(c, d)$ by the vector from $M^{\prime}$ to $M$. Hence

$$
\begin{aligned}
D & =T \cdot \text { abs. value of }\left|\begin{array}{cc}
\left(-R \sin \theta+R^{\prime} \cos \theta\right)\left(R \cos \theta+R^{\prime} \sin \theta\right) \\
(-k+\cos \psi) & \sin \psi
\end{array}\right| \\
& =T\left|D^{*}\right|
\end{aligned}
$$

where

$$
\begin{aligned}
D^{*}= & +R(-\sin \theta \sin \psi-\cos \theta \cos \psi) \\
& +R^{\prime}(\cos \theta \sin \psi-\sin \theta \cos \psi) \\
& +k\left(R \cos \theta+R^{\prime} \sin \theta\right) \\
= & -R \cos \phi+R^{\prime} \sin \phi+k\left(R \cos \theta+R^{\prime} \sin \theta\right) .
\end{aligned}
$$

Not all couples $(\theta, \phi)$ may occur as contacts. The relative velocity of particle $B$ with respect to $A$ is

$$
-k+\cos \psi, \sin \psi
$$

A contact is possible at $(\theta, \phi)$ if and only if the particle $B$ enters the curve $R(\theta)$ from without. Since the curve $R=\omega(\theta)$ is convex by hypothesis, this will occur when and only when the angle, from the tangent to the curve at $\theta$ to the relative velocity vector of $B$ is between zero and $\pi$. But this is exactly the condition that $D^{*}>0$. We conclude that

$$
f(\theta, \phi)=c\left(D^{*}+\left|D^{*}\right|\right),
$$

where $c$ is a constant defined by

$$
\int_{0}^{2 \pi} \int_{0}^{2 \pi} c\left(D^{*}+\left|D^{*}\right|\right) d \theta d \phi=1
$$

This is the desired result.

Dr. Charles Kittell proposed a somewhat more specialized version of the problem 
of this paper to the author, who gave the solution in 1945 while a member of the Statistical Research Group of Columbia University, which was working under contract with the National Defense Research Committee of the Office of Scientific Research and Development.

\title{
A COMMENT ON F. N. FRENKIEL'S NOTE “ON THIRD-ORDER CORRELATION AND VORTICITY IN ISOTROPIC TURBULENCE"*
}

\author{
By G. K. BATCHELOR ANd A. A. TOWNSEND (Cambridge University)
}

The short paper by Mr. F. N. Frenkiel [this Quarterly, 6, 86-90 (1948)] calls for some comment. Mr. Frenkiel makes certain assumptions about the correlation functions and derives relations for the decay of energy and mean-square vorticity in isotropic turbulence. These relations are not in agreement with measurements which we have already published. ${ }^{1}$ Mr. Frenkiel concludes that "if account is taken of probable inaccuracies in the experimental data (in Ref. 1), it appears that the agreement (with his deductions) may be satisfactory after all." In answer to this suggestion, we make the following points.

(1) In our opinion there is no possibility that experimental error would account for the discrepancies between our data and Mr. Frenkiel's results. He finds $d \lambda^{2} / d t=7 \nu$, whereas according to our measurements, $d \lambda^{2} / d t=10 \nu$ with a standard deviation of less than $0.4 \nu$. Our measurements were corrected for the effect of finite length of the recording hot-wire but, in any case, the correction leaves $d \lambda^{2} / d t$ unaltered provided the wire length is small enough to be comparable with $\lambda$. Mr. Frenkiel also suggests lack of isotropy as a possible source of error. The presence of isotropy to a sufficient approximation seems to us to have been checked very well by the consistency of measurements of $\bar{u}$ and $\lambda$ (which are related by the energy equations for isotropic turbulence) and of $\overline{u^{2}}, \lambda, f_{0}^{\text {iv }} \lambda^{4}$ and $k_{0}^{\prime \prime \prime} \lambda$ (which are related by the vorticity equation).

(2) There is a strong body of theory ${ }^{2}$ concerning the mechanism of turbulence at high Reynolds numbers, which has been shown ${ }^{3}$ to link up very satisfactorily with the measurements of Ref. 1. Any contrary results, such as that given by Mr. Frenkiel for the variation of $k_{0}^{\prime \prime \prime} \lambda^{3}$ during decay, would therefore need strong experimental and theoretical backing.

(3) Mr. Frenkiel gives no reasons to support his assumption that the correlation curve is self-preserving outside the (small) region in which $r$ is comparable with $\lambda$. Indeed, it leads him into inconsistencies. Later in his paper he assumes, in agreement with our measurements, that $f_{0}^{\mathrm{i} \nu} \lambda^{4}$ is constant during decay. This implies that the correlation curve is also self-preserving for small values of $r$ and it has now been assumed in effect that $f(r)$ is completely self-preserving. Equation (6) of his paper then leads to the conclusion that $\lambda\left(\overline{u^{2}}\right)^{1 / 2} / \nu$ is constant during decay, whereas his published result is that this quantity varies as $t^{-3 / 14}$. Again, he is willing to assume $f_{0}^{\text {iv }} \lambda^{4}$ independent of $t$, and any physical basis for this assumption would seem to lead also to $k_{0}^{\prime \prime \prime} \lambda^{3}$ being independent of $t$ (as our measurements show), but Mr. Frenkiel finds $k_{0}^{\prime \prime \prime} \lambda^{3}$ is proportional to $t^{3 / 14}$.

${ }^{*}$ Received June 16, 1948.

${ }^{1}$ G. K. Batchelor and A. A. Townsend, Proc. Roy. Soc. (A) 190, 534 (1947).

${ }^{2}$ A. N. Kolmogoroff, C. R. Acad. Sci. U.R.S.S. 30, 301 (1941) and 32, 16 (1941).

${ }^{3}$ G. K. Batchelor, Proc. Camb. Phil. Soc. 43, 534 (1947). 